\title{
¿Por qué hago evaluación formativa y compartida y/o evaluación para el aprendizaje en EF? La influencia dela formación inicial y permanente del profesorado \\ Why do I do Formative and Share Assessment and/or Assessment For Learning in Physical Education? The influence of Teacher Education \\ Emilio Barrientos Hernán, Víctor Manuel López Pastor, Darío Pérez-Brunicardi \\ Universidad de Valladolid(España)
}

Resumen. La finalidad de este artículo es analizar si una muestra intencionada de profesores y maestros de educación física(EF) utilizan sistemas de evaluación formativa y compartida (EFyC) y/o de evaluación para el aprendizaje (EPA) en sus clases y la influencia que ha podido tener su formación inicial y permanente (FIP y FPP) en el sistema de evaluación que realizan actualmente. Se llevan a cabo entrevistas en profundidad a una muestra de 3 maestros de Primaria y 1 profesor de Secundaria, seleccionados en base a tres criterios. Los resultados señalan que la aplicación de sistemas de EFyC y/o EPA mejora la implicación y participación del alumnado en su propio aprendizaje y favorecen la regulación del proceso de enseñanza y aprendizaje; los alumnos se toman más en serio el área de EF y los maestros organizan mejor el proceso de enseñanza. Por otro lado, la FIP y FPP parecen ayudar a ir modificando las prácticas evaluativas del profesorado que busca un sistema de evaluación más coherente con sus creencias educativas, valorando especialmente la participación en actividades de FPP que les aporten experiencias útiles y aplicables en su práctica. Palabras clave: Evaluación Formativa, Evaluación Para el Aprendizaje, Formación del Profesorado, Educación Física.

Abstract. The study main goal is to analyze if an intentional sample of Physical Education (PE) teachers use Formative and Share Assessment (F\&SA) and/or Assessment For Learning (AFL) in their lessons and, and if they have been influenced by their Teacher Education (TE) to carry out their current assessment systems.In-depth interviews were conducted with four PE teachers: three from Primary Education and one from Secondary Education, the four having been selected through three criteria. On one hand, the application of F\&SAand/or AFL improves students' involvement and participation in their own learning and regulate the teaching and learning processes; students give PE subject more importance and teachers organize the teaching process better. On the other hand, the TE seems to modify the assessment practices of the teachers. They look for an assessment system more coherent with their educational beliefs, assessing in a very positive way, the participation in in-service TE that provides them with useful and applicable experiences to their practice.

Keywords: Formative Assessment, Assessment For Learning, Teacher Education, Physical Education.

\section{Introducción}

\section{Modelos de evaluación en Educación Física (EF)}

En los últimos años se ha investigado en profundidad sobre las competencias docentes necesarias en los procesos de enseñanza-aprendizaje, especialmente ligado a los estudios universitarios de formación inicial del profesorado (FIP). Un grupo importante de estas competencias profesionales son las relacionadas con el tema de la evaluación; tanto las que hacen referencia a la evaluación del aprendizaje del alumnado, como a la evaluación del desempeño docente y de los procesos de enseñanza-aprendizaje en el aula. Para Perrenoud (2004) la evaluación es una competencia docente básica y, también, una de las más costosas y tediosas de la función docente, que genera miedos y reticencias, hasta el punto de que se trata de uno de los aspectos en el que más cuesta cambiar e innovar (Zabalza, 2003). Según Morgan \& Hansen(2007), es la competencia en la que los maestros de educación primaria se sienten menos capaces en su práctica docente.

Por otra parte, parecen existir dos modelos opuestos a la hora de entender y llevar a cabo la evaluación en EF: (a) los sistemas que se aproximan al modelo: «tradicional-sumativo»; y, (b) los sistemas de evaluación «alternativa», que se aproximan al modelo de evaluación formativa y compartida (EFyC) y/o evaluación para el aprendizaje (EPA) (López-Pastor, Kirk, Lorente, MacPhail \& Macdonald, 2013). El modelo de evaluación tradicional-sumativo en EF suele centrarse en cómo obtener la calificación de los alumnos al final de un trimestre o curso y suele predominar la utilización de test de condición física y/o habilidad motriz. Por lo tanto, la nota final del alumno procede de los resultados obtenidos en dichos test, o al menos un porcentaje importante de la misma (López-Pastor, 1999; López-Pastor etal., 2013; MacPhail \& Murphy, 2017).

Fecha recepción: 02-07-18. Fecha de aceptación: 27-12-18 Víctor Manuel López Pastor vlopez@mpc.uva.es

\section{Evaluación formativa, feedback y feedforward}

Los modelos de evaluación formativa defienden que la finalidad principal de todo proceso de evaluación debe ser la de mejorar los procesos de enseñanza-aprendizaje que tienen lugar. Dicho de otra manera, todo proceso de evaluación que sirva para que el alumnado aprenda más y para que el profesorado pueda perfeccionar su práctica docente. En este modelo la finalidad no es calificar al alumno, sino disponer de información que permita saber cómo ayudar al alumnado a mejorar y aprender más, a la vez que sirve para que los profesores realicen su trabajo cada vez mejor (López-Pastor, 2006; López-Pastor et al., 2013; MacPhail \& Murphy, 2017; Tolgfors \& Öhman, 2015). Según la mayoría de estos autores, la evaluación en EF debería ser también «compartida»; dado que debería fomentar la participación del alumnado en los procesos de evaluación, dado que también están implicados en los procesos de enseñanza-aprendizaje que tienen lugar en el aula. Entendida de esta manera, la evaluación debe ser más un diálogo y una toma de decisiones mutuas y/o colectivas que un proceso individual e impuesto. Las técnicas para llevar a cabo procesos de evaluación compartida en EF suelen ser: la autoevaluación, la co-evaluación y la evaluación dialogada. En López-Pastor (2006) y López-Pastor y PérezPueyo (2017) pueden encontrarse numerosas experiencias y ejemplos de técnicas e instrumentos sobre EFyC en EF en todas las etapas del sistema educativo (educación infantil, primaria, secundaria y universidad), además de su fundamentación teórica.

Parece existir una relación muy estrecha entre evaluación formativa y los procesos de feedback y feedforward. Cuando el feedback es utilizado dentro de un sistema de evaluación formativa puede denominarse como «Feedback Formativo», en el que además de dar información para que los aprendices cubran el espacio existente entre el actual nivel de aprendizaje y el que se desea que consigan, implicaría que los aprendices interiorizarán esa información y la utilizaran de manera constructiva en sus aprendizajes (Taras, 2005). En cambio, el término «Feedforward» tiene dos significados (Dulama \& Iloran, 2016): (a) el primero está centrado en las respuestas que dan los alumnos al feedback dado por el maestro; en el sentido de que el alumno tendría que ser 
consciente de la meta del feedback e interpretarlo de tal manera que sería capaz de cerrar el hueco existente entre su nivel actual de aprendizaje y el nivel de desarrollo que se desea que consiga (Koen, Bitzer, \& Beets, 2012); (b) el segundo hace referencia al uso que el alumno hará en el futuro de los aprendizajes realizados previamente con el feedback dado por el profesor (Gibbs \& Simpson, 2004; Koen et al., 2012; Murtagh \& Baker, 2009); por lo tanto, se centraría en resolver problemas y no en corregir errores. En este sentido, los alumnos conocen sus expectativas futuras y se convierten en participes del proceso de enseñanza.

Por tanto, el «Feedforward» se refiere a la aplicación o transferibilidad que un estudiante puede hacer en el futuro del feedback recibido por el profesor. En este sentido, el que el profesor deje claras sus expectativas y estándares de aprendizaje puede servir como ayuda para el alumno (Baker \& Zuvela, 2013). Goldsmith (2008) explica que la principal diferencia entre «feedback» $\mathrm{y}$ «feedforward» es que el segundo está enfocado al futuro, mientras que el primero está enfocado al pasado; esto es, está enfocado a resolver problemas futuros, más que a corregir errores ya cometidos. Por ello puede ser más eficiente que el feedback, ya que ayuda a que el alumno tenga más autonomía en la gestión de sus propios aprendizajes.

Evaluación formativa y evaluación para el aprendizaje en EF Algunas investigaciones muestran que el uso de sistemas de evaluación formativa mejora el aprendizaje de los alumnos (Black \& Wiliam 1998). Algunos autores defienden que la evaluación formativa es la llave para mejorar en el proceso de consecución de cualquier área y las evidencias muestran «que funciona» a lo largo de Europa (Wiliam \& Leahy, 2015). En cambio en EF se pueden encontrar resultados diferentes. Por un lado, algunas investigaciones internacionales centradas en el impacto de la evaluación formativa en EF parecen ofrecer resultados limitados (Hay, 2006; MacPhail \& Halbert, 2010; MacPhail \& Murphy, 2017) mientras que otros estudios parecen indicar que el uso de una evaluación formativa parece mejorar la calidad de los procesos de enseñanza y aprendizaje en EF en primaria e impacta de manera positiva en la percepción de alumnos y maestros sobre la EF (López-Pastor, 1999, 2006; Ní Chróinín \& Cosgrave, 2013) hasta el punto de que diferentes autores consideran que la evaluación formativa es una parte integral del proceso de enseñanza-aprendizaje en EF (Richard \& Godbout, 2000; MacPhail \& Halbert, 2010). Por otra parte, también pueden encontrarse estudios que demuestran que cuando se trabaja con modelos pedagógicos de aprendizaje cooperativo en EF, la implementación de una evaluación coherente con dicho modelo ayuda a mejorar el aprendizaje del alumnado y la calidad educativa de los procesos de enseñanza-aprendizaje que se desarrollan (López-Pastor et al., 2008). Por último, otros estudios indican que cuando los alumnos son partícipes de los criterios de evaluación de su propio aprendizaje ayuda a que su aprendizaje aumente y estén más implicados (Svennberg et al., 2014; López-Pastor, 1999, 2006; Herranz \& López-Pastor, 2014).

Revisando la literatura internacional especializada (Casbon \& Spackman, 2005; Hay, 2006; Leirhaug \& MacPhail, 2015; Lorente \& Kirk, 2013; MacPhail \& Halbert, 2010; Mac Phail \& Murphy, 2017; Ní Chróinín \& Cosgrave 2013; Richard \& Godbout, 2000; Tolgfors \& Öhman, 2015), puede comprobarse que el término «Evaluación Formativa» en EF ha evolucionado hacia otros más centrados en el proceso de aprendizaje del alumnado, como: «evaluación alternativa» (EA),»evaluación para el aprendizaje» (EPA), «evaluación orientada al aprendizaje» (EOA) o «evaluación auténtica» (EA) (López-Pastor et al., 2013).

En un primer momento, MacPhail \& Halbert (2010) consideran que el concepto de EPA se caracteriza por cuatro principios, pero posteriormente diferentes autores lo amplían a cinco (Leirhaug \& MacPhail, 2015; Tolgfors \& Öhman, 2015): (1) clarificar y compartir las intenciones de aprendizaje con los estudiantes; (2) innovar en la efectividad de las conversaciones en clase, tareas y actividades que muestren evidencia del aprendizaje; (3) proveer feedback que ayude a avanzar al alumno; (4) activar a los estudiantes como recursos de aprendizaje de unos para otros; (5) activar a los estudiantes como responsa- bles de su propio aprendizaje. En la literatura especializada pueden encontrarse diferentes estudios sobre la aplicación del EPA en EF. Por ejemplo, Leirhaug \& MacPhail (2015) realizan un estudio en Noruega con 3 maestros de EF para comprobar qué tipo de evaluación estaban llevando a cabo y sí esta era de tipo EPA. Encuentran que las prácticas evaluativas de estos maestros estaban lejos de cumplir los cinco principios señalados. En una línea similar, Leirhaug \& Annerstedt (2016) investigan si en las clases de EF en Noruega se está utilizando un currículo enfocado al EPA, dado que las autoridades educativas lo han introducido en la normativa. Los estudiantes señalan que las prácticas de EF no reflejan los principios del EPA y que la manera de entender esos principios por parte de los profesores puede variar dependiendo de cómo los interpreten. Esto podría indicar que dicha normativa no está siendo aplicada de manera adecuada en las clases de EF. Por su parte, Borghouts, Slingerland \& Haerens (2016) realizan un estudio en Holanda con una muestra de 206 maestros de EF para conocer la importancia de implementar una EPAen sus clases. La realidad muestra que suele haber poca coherencia entre los objetivos de aprendizaje que se plantean para los alumnos y la evaluación utilizada; por ejemplo, el 81\% evalúa la condición física como un aspecto básico, aunque no aparece en los objetivos. Los maestros afirman que consideran el EPA importante, pero sus prácticas evaluativas no parecen cumplirlo.

Por otra parte, uno de los conceptos más interesantes que están ligados a toda la red nomológica de «evaluación alternativa y formativa» es el de «evaluación auténtica» (EA). López-Pastor et al. (2013) consideran la EA como un sistema de evaluación estrechamente relacionado con la evaluación formativa. El concepto de EA en EF hace referencia al hecho de que las técnicas, instrumentos y actividades de evaluación estén claramente aplicados en situaciones, actividades y contenidos reales del aprendizaje. Se opone, por tanto, a las situaciones puntuales y artificiales de evaluación, alejadas de la práctica real o de la aplicación real de dichos conocimientos (Desrosiers, Genet-Volet \& Godbout, 1997; López-Pastor et al., 2013; Mintah, 2003; Oslin, Mitchell \& Griffin, 1998; Richard \& Goudbout, 2000).

La tendencia a reproducir como profesores de educación física lo que han vivido como alumnos durante su formación inicial

Desgraciadamente, en EF todavía siguen demasiado implantados los modelos tradicionales de evaluación-calificación, basados en la realización de test de condición física o habilidad motriz, con la única o principal finalidad de calificar al alumnado (López-Pastor, 1999, 2006; López-Pastor et al., 2013). Sin embargo, las nuevas tendencias actuales están centradas en desarrollar sistemas de evaluación más orientados al aprendizaje del alumnado. En la Formación Inicial del Profesorado(FIP) de EF hace tiempo que existe preocupación por implantar y desarrollar nuevos sistemas de evaluación, más orientados hacía enfoques de EFyC, EPAy EA(Fernández-Balboa, 2006; López-Pastor, 2006, 2008; LópezPastor \& Sicilia, 2017; Lorente \& Kirk, 2013), así como la importancia de fijar un modelo de competencias profesionales (Gomez, Del Valle \& De la Vega, 2018).

Algunos estudios coinciden en que el alumnado que utiliza sistemas de EFyC en FIP, en los que el feedback se da más frecuentemente, obtiene un mayor rendimiento académico que el alumnado que sigue los sistemas y vías basados en examen final (Fraile-Aranda, López-Pastor, Castejón-Oliva \& Romero-Martín, 2013; López-Pastor, 2008; Lorente \& Kirk, 2013; Romero-Martín, Fraile-Aranda, López-Pastor \& Castejón-Oliva, 2014).

Pero en cambio, parece que trasferir estos planteamientos a la enseñanza de la EF en primaria y secundaria tiene más dificultades y obstáculos. Mintah (2003) explicaba que las principales razones de los maestros de EF para no utilizar la EA parecen ser que requiere más tiempo y que es difícil de llevar a cabo cuando se tienen pocas horas de EF a la semana con cada grupo de alumnos y, sobre todo, que el profesorado no está suficientemente formado para la utilización de la EA. Morgan \& Hansen (2007) encontraron que para los maestros de EF en primaria la evaluación es la competencia en la que se sienten menos capaces en su práctica docente. En este sentido Ní Chróinín \& Cosgrave 
(2013) encuentran que los sistemas de evaluación formativa son evitados por los maestros de EF en primaria.

En Hamodi, López-Pastor \& López (2017) realizan un estudio sobre la posible trasferencia entre los sistemas de EFyC vividos durante la FIP y su posterior aplicación en la práctica en la escuela. Los resultados indican que sí existe dicha trasferencia, aunque surgen obstáculos y dificultades, sobre todo en forma de diferentes tipos de resistencias por parte de algunos compañeros, estudiantes y padres. Estos maestros valoran positivamente haber tenido experiencias de evaluación formativa durante su FIP, a pesar de haber sido pocas. También afirman que dichas experiencias les han influido positivamente a la hora de intentar desarrollar ese tipo de sistemas de evaluación cuando comenzaron a trabajar como profesores de EF en primaria. Pueden encontrarse algunas referencias de estudios autobiográficos que explican porque algunos profesores y maestros de EF comienzan a aplicar sistemas de EFyC en sus aulas, en contra de la tradición y la formación recibida (Córdoba, 2015; López-Pastor, 1999, 2006; Molina \& López, 2017). Todos ellos muestran la enorme influencia que parecen tener los procesos de formación permanente del profesorado, especialmente cuando están asociados a procesos de desarrollo profesional ligados a grupos colaborativos de investigación-acción (Córdoba et al., 2016; López-Pastor et al., 2016; Pedraza \& López-Pastor, 2015; Lorente \& Kirk, 2013).

Sin embargo, es muy difícil encontrar estudios sobre las razones por las que algunos profesores y maestros de EF ponen en práctica sistemas de EFyC y/o EPA. Por ello, los objetivos de investigación que hemos fijado para este estudio son:

1- Analizar si una muestra intencionada de profesores de EF de educación obligatoria (primaria y secundaria) utiliza sistemas de EFyC y/o de EPA en sus clases.

2-Estudiar la influencia que ha podido tener su formación inicial y/ o permanente en el desarrollo de sistemas de EFyC en EF en la educación obligatoria.

\section{Metodología}

\section{Diseñoymuestra}

El diseño es un estudio multicaso, basado en la utilización de metodología cualitativa, concretamente entrevistas en profundidad a cuatro profesores de EF: tres de Educación Primaria y uno de Secundaria. Se trata de una muestra intencional, de cuatro profesores y profesoras de EF que cumplen los criterios establecidos para formar parte de la muestra. Los entrevistados fueron seleccionados de acuerdo a los siguientes criterios: (a) compartir con los estudiantes los objetivos de aprendizaje y los criterios de evaluación a utilizar en la «unidad didáctica» (UD) o curso; (b) implicar al alumnado en la evaluación de su propio aprendizaje; (c) facilitar feedback a los estudiantes, que les ayude a entender que deben hacer para mejorar su aprendizaje.

En la tabla 1 explicamos brevemente las características profesionales de cada uno.

\section{Técnica de obtención de datos}

Se han llevado a cabo entrevistas en profundidad a los cuatro pro- fesores que constituyen la muestra intencional. Las entrevistas se inician con un primer bloque de preguntas relacionadas con características profesionales de los entrevistados (ver tabla 1). La segunda parte se centra en aspectos clave del objeto de investigación, como: qué sistema de evaluación llevan a cabo actualmente, cómo registraban la información recogida durante el proceso y los motivos que les han llevado a realizar ese tipo de evaluación. Todas las entrevistas comienzan proporcionando las garantías de anonimato y confidencialidad de las respuestas proporcionadas, además se solicita autorización para poder grabar la entrevista.

\section{Análisis de datos}

Para analizar los datos se ha llevado a cabo, en primer lugar, un proceso de transcripción de las entrevistas. Posteriormente se ha realizado un proceso de codificación y categorización de los datos recogidos. Se ha optado por no utilizar programas informáticos para su análisis. En la tabla 2 se presenta el sistema de categorías y subcategorías que emerge del análisis de datos y que hemos utilizado como estructura básica para presentar los resultados.

\begin{tabular}{|c|c|}
\hline Categorías & Subcategorías \\
\hline $\begin{array}{l}\text { 1-Utilización de EFyC y/o } \\
\text { EPA en las clases de EF. }\end{array}$ & $\begin{array}{l}\text { 1.1- Compartir los objetivos de aprendizaje y los criterios de } \\
\text { evaluación con los estudiantes. } \\
\text { 1.2- Implicar al alumno en la evaluación de su aprendizaje. } \\
\text { 1.3- Uso de Feedback que mejore el aprendizaje de los alumnos. }\end{array}$ \\
\hline $\begin{array}{l}\text { 2- Papel de la FIP en la } \\
\text { realización o no de prácticas } \\
\text { de EFyC y/o EPA. }\end{array}$ & $\begin{array}{l}\text { 2.1- Evaluación vivida durante la FIP y evolución hasta el tipo } \\
\text { de evaluación actual. } \\
\text { 2.2- Formación recibida/realizada hasta la situación actual. }\end{array}$ \\
\hline
\end{tabular}

\section{Resultados y discusión}

Este apartado se ha organizado en función del sistema de categorías y subcategorías presentado en la Tabla 2. Dado que la investigación es de corte cualitativo, hemos unido en un mismo apartado la presentación de resultados y la discusión. Al final de cada cita literal ofrecemos información del origen de los datos según la siguiente clave: la letra «E» indica entrevista; los números «1, 2, 3y 4» indican el orden del profesor al que se realiza la entrevista.

Utilización de Evaluación Formativa y Compartida y/o Evaluación para el Aprendizaje en las clases de Educación Física

Todos los entrevistados llevan a cabo el primer criterio, que hacía referencia a compartir los criterios de evaluación y los objetivos de aprendizaje con los alumnos. Con ello, los alumnos son más conscientes de la evaluación que van a recibir y de las metas que han de alcanzar en su proceso de aprendizaje:

Al alumno le es más fácil seguirte siél sabe cuáles son las condiciones, las reglas del juego. Pero si no dices nada, no saben en qué aspectos concretos deberían haber trabajado. Por ejemplo, si ellos tienen las reglas del juego al principio de la UD pues saben en que se tienen que fijar y se centran en la mejoría de los aspectos que tú quieres (E3).

Antes de bajar al patio, en clase, los alumnos escribían en su

\begin{tabular}{|c|c|c|c|c|}
\hline & Profesor 1 & Profesor 2 & Profesor 3 & Profesor 4 \\
\hline Edad & 36 & 38 & 43 & 41 \\
\hline Formación inicial & INEF-CAFYD & INEF-CAFYD & Maestro de EF & Maestro de EF \\
\hline Formación complementaria & Doctor & Maestro de EF & $\begin{array}{l}\text {-Pedagogía } \\
\text {-Cursos de Doctorado }\end{array}$ & $\begin{array}{l}\text {-Pedagogía } \\
\text {-Doctor (tesis sobre EFyC) }\end{array}$ \\
\hline $\begin{array}{l}\text { Experiencias de EFyC } \\
\text { durante su FIP }\end{array}$ & Ninguna & Ninguna & Ninguna & Sí, en algunas asignaturas \\
\hline Formación permanente & $\begin{array}{l}\text {-Forma parte del grupo de trabajo que } \\
\text { usan EFyC. } \\
\text {-Contacto con gente que utiliza EFyC. } \\
\text {-Lectura de libros y artículos. } \\
\text {-Asistencia a congresos de evaluación. }\end{array}$ & $\begin{array}{l}\text {-Contacto con grupo de trabajo y colegas } \\
\text { que usan EFyC. } \\
\text {-Lectura de libros y artículos relacionados } \\
\text { con la temática. } \\
\text {-Asistencia a congresos de evaluación y } \\
\text { metologías alternativas. }\end{array}$ & $\begin{array}{l}\text {-Forma parte del grupo de trabajo y colegas } \\
\text { que usan EFyC. } \\
\text {-Lectura de revistas de EF. } \\
\text {-Asistencia a congresos de evaluación. }\end{array}$ & $\begin{array}{l}\text {-Tesis doctoral sobre EFyC en EF en } \\
\text { primaria. } \\
\text {-Cursos de formación permanente. } \\
\text {-Participa en seminario de EFyC en } \\
\text { educación. } \\
\text {-Asistencia a congresos. }\end{array}$ \\
\hline $\begin{array}{l}\text { Participación en GT o en } \\
\text { proyectos de innovación } \\
\text { docente relacionados }\end{array}$ & $\begin{array}{l}\text { GT Segovia. } \\
\text { GT Avila. }\end{array}$ & GT de Segovia. & GT de Segovia. & Seminario EFyC en educación, \\
\hline Experiencia docente & $\begin{array}{l}3 \text { años en la Universidad y } 2 \text { años en } \\
\text { secundaria. }\end{array}$ & 4 años en secundaria y 8 en primaria. & 20 años de EF en Primaria. & 12 años en EF y otras especialidades. \\
\hline $\begin{array}{l}\text { Cuántos cursos utilizando } \\
\text { sistemas de EFyC o similares }\end{array}$ & $\begin{array}{l}\text { En secundaria } 2 \text { años. } \\
\text { s }\end{array}$ & 8 años desde que lleva en Primaria. & 15 años en Primaria. & 12 años en Primaria. \\
\hline ¿Qué ratio de alumnos tiene? & ? Entre 16 y 24. & Entre 18 y 22. & Unos 25 por clase. & Unos 17-18 alumnos por clase. \\
\hline
\end{tabular}


cuaderno los conceptos clave de la UD, por ejemplo: en la UD de Lanzamientos, los conceptos claves que vamos a trabajar son....y les decía las 5-6 cosas importantes en las que nos ibamos a centrar a lo largo de la UD. El preparar y organizar la sesión en el aula hacia que los niños ya salieran preparados, supieran que íbamos a hacer y no se dispersaran. (E4).

Dentro de este tipo de procesos, parece ser bastante habitual la utilización de rúbricas de evaluación, y la facilitación de dichas rúbricas al alumnado al principio del trimestre o de la unidad didáctica. También enun caso surge la temática dela calificación, de cómo facilitar al alumnado los criterios de calificación desde el principio, dado que es un tema que les preocupa mucho:

Las rúbricas de autoevaluación se valoran al final del trimestre pero se entrega al inicio del mismo, para que los alumnos sepan cuáles son los aspectos importantes que tienen que aprender ellos, yo creo que es lo más importante para ellos (E2).

Les explico a los alumnos al inicio de curso los criterios de calificación y les pregunto si les parecen bien, pero normalmente nadie dice nada y no se implican mucho. Eso sí, cuando ven que va a llegar la nota ya les preocupa mucho (fin del trimestre). Además, en la rúbrica los alumnos saben perfectamente lo que les voy a evaluar, lo que les pido. Yo creo que así, se ajusta más al objetivo de aprendizaje (E1).

Como hemos visto, una parte esencial de la EFyC es que el alumnado sea consciente y partícipe de los objetivos de aprendizaje que se desea que alcancen (López-Pastor \& Pérez-Pueyo, 2017). Además, los resultados recogidos en las entrevistas muestran que informar a los alumnos de los criterios de evaluación al inicio de un nuevo proceso de aprendizaje (UD, trimestre, sesión) tiene aspectos positivos: (1) el feedback que reciben está relacionado con los aspectos clave a aprender; (2) el alumnado se toma más en serio la EF y mejora el clima general dentro de las sesiones de clase; lo que coincide con los resultados obtenidos en otros estudios sobre la temática (Herranz \& López-Pastor 2014; MacPhail \& Halbert 2010; NíChróinín \& Cosgrave 2013; Svennberg et al., 2014). Por ejemplo, Taras (2005) defiende que el «feedback formativo» implica que los aprendices interioricen la información dada y que la utilicen de manera constructiva en sus aprendizajes, y diferentes autores defienden el interés en avanzar hacía procesos de «Feedforward», en los que es fundamental que el alumnado conozca los objetivos de aprendizaje y se conviertan en participes del mismo (Baker \& Zuvela, 2013; Gibbs \& Simpson, 2004; Goldsmith, 2008; Dulama \& Iloran, 2016; Koen et al., 2012; Murtagh \& Baker, 2009).

Los entrevistados afirman utilizar diversas estrategias para que el alumno pueda implicarse en la evaluación de su aprendizaje. Algunos de los instrumentos utilizados son: cuestionarios, rúbricas, grabaciones, escalas descriptivas y portafolios. En todos ellos son los alumnos quienes autoevalúan su proceso y, en algunos casos, se utilizan para coevaluar los aprendizajes de otros compañeros:

Las grabaciones realizadas por profesor y alumnos se usan para heteroevaluar, coevaluar y autoevaluar. Para ello, utilizo la plataforma del Dropbox para el intercambio de material (allí los alumnos pueden subirlo y compartirlo directamente). También, utilizo la rúbrica para que los alumnos se coevalúen y autoevalúen, ya que me parece muy clara para el alumno, sabiendo perfectamente lo que tú les pides, lo qué se va a evaluar y se ajusta mejor a los objetivos de aprendizaje que me propuse que alcanzaran los alum$\operatorname{nos}(E 1)$.

Cuando quiero hacer una coevaluación con los niños considero que una escala descriptiva va muy bien, sobre todo para iniciarse en lo que es el proceso de evaluación. Si tienes los ítems marcados y cada uno de ellos tiene sus subescalas $(a, b, c)$, orientas la conducta del alumno y les resulta más fácil conocer que observar (poniendo una cruz en lo que ellos crean) (E2).

Los datos mostrados señalan que los procesos de autoevaluación, coevaluación y evaluación compartida son clave para implicar al alumnado en su evaluación, coincidiendo con lo ya explicado por otros autores y por lo realizado en otras experiencias (Herranz \& López-
Pastor, 2014, 2017; López-Pastor, 2006; López \& Gutiérrez, 2018; MacPhail \& Murphy, 2017; Tolgfors \& Öhman, 2015). Como puede comprobarse, estos profesores hablan de diferentes instrumentos y procesos de evaluación, pero haciendo un énfasis especial en el uso de escalas descriptivas o «rúbricas». En López-Pastor (2006, 2009) y López-Pastor y Pérez-Pueyo (2017) pueden encontrarse descripciones detalladas de los diferentes instrumentos de evaluación y como a veces el mismo instrumento es denominado de dos formas diferentes, como es el caso.

Los resultados también muestran que estos profesores utilizan la autoevaluación a lo largo de todo el proceso educativo, y no solo de manera puntual.

Los alumnos tienen un cuestionario que utilizan en cada UD ( $a$ veces al final, otras en el medio y al final, y en el inicio, en el medio y al final). Lo bueno de pasarlo al inicio es que les «entra» un poco más. Lo utilizo como instrumento de autoevaluación al final de la UD (ese momento seguro) y a veces también al inicio y al final de

la UD. En ellos, los alumnos tienen una escala y van marcando el progreso que han obtenido, desde «es poco» hasta «mucho» (es poco, un poco, bastante o mucho). El cuestionario se divide en dos, una primera parte donde los alumnos ponen cruces en cada una de las afirmaciones según la escala citada anteriormente y otra segunda parte con preguntas de respuesta abierta (E3).

Tal como algunos estudios indican, de esta forma se aumenta el potencial formativo de la participación del alumnado en la evaluación (López-Pastor et al., 2013), e incluso algunos autores señalan la autoevaluación como uno de los cinco pilares principales para producir una evaluación formativa efectiva (Wiliam \& Leahy, 2015). Dentro de este mismo enfoque, Black \& Wiliam (1998) afirman que es preferible que existan procesos de autoevaluación durante todo el aprendizaje, para que los estudiantes puedan revisar su trabajo continuamente y no sólo al final.

Además, uno de los profesores entrevistados explica de forma clara la conexión entre los dos criterios analizados hasta el momento: compartir con el alumnado los criterios de evaluación y los objetivos de aprendizaje, e implicarles posteriormente en el proceso de evaluación, especialmente al final de la misma, completando el proceso de pasar de una autoevaluación final a una evaluación compartida (López-Pastor et al., 2006):

Cada día al llegar al aula, ponía en la pizarra en los primeros 5-10 minutos los aspectos que íbamos a trabajar, como estructuraba la sesión y ponía las actividades que hacíamos en cada parte. Después usaba esa información en la autoevaluación de la UD, con respecto a los objetivos que yo previamente les había dado, les decía: los conceptos clave de esta UD son estos, y ellos en un cuaderno escribían, por ejemplo: «UD de Lanzamientos, chicos, los conceptos claves que vamos a trabajar en esta UD son....y les decía los 5-6 cosas importantes en las que nos íbamos a centrar a lo largo de la UD». Al final de la UD se pasaba un cuestionario de autoevaluación en la que los ítems eran conceptos clave que habíamos copiado inicialmente en la UD. Ellos tenían que valorar los aprendizajes, ellos ya sabían lo que se les iba a preguntary después de eso, hacíamos una entrevista personal (E4).

Los resultados demuestran que todos los entrevistados cumplen el tercer criterio, que hacía alusión a dar un feedback que les ayude a los alumnos a avanzar en sus aprendizajes, ofreciendo tres tipos: gran grupo, pequeño grupo e individuales:

El Feedback puede ser inmediato o posterior y principalmente es verbal. Ano ser que hagan producciones, que yo corrija y después les doy el feedback. A veces lo doy en grupo (si no lo ha entendido bien lo que hay que hacer un porcentaje alto de la clase) y a veces individual o en grupos pequeños (si es una cosa puntual) (E1). Doy el feedbacken las paradas de reflexión, a nivel general, y si veo algo extraordinario, voy al grupo en cuestión y les hago a ellos la observación; esto sería de manera puntual (E3).

También existen matices y diferencias sobre el cómo y cuándo darlo, y la importancia de intentar integrarlo en el proceso de aprendiza- 
je de la forma más ágil posible, aunque a veces sea necesario realizar pequeñas paradas de reflexión-acción:

Cada vez que terminamos una UD les doy pequeños feedback de cómo va el proceso. Los realizo casi todos grupales (asambleas), ya que individualmente no hay casi tiempo. Además, cuando veo que hay un error común, lo ideal sería realizar una parada y dar a los alumnos un feedback, para que cambien ciertos aspectos. En cuanto un alumno realiza una acción muy buena, nombrarle para que siga en la misma línea, intentando parar lo menos posible lo que es la clase (E2).

Doy feedback al alumno en entrevistas individuales muy rápidas o correcciones inmediatas en la pista sobre aspectos clave de lo que están haciendo. También a veces se para la actividad, se hace una pausa, se explica y seguimos (E4).

Estos aspectos coinciden con lo señalado por diferentes autores, que consideran que la evaluación formativa es una parte integral del proceso de enseñanza-aprendizaje en EF (Richard \& Godbout, 2000; MacPhail \& Halbert, 2010). En todos los casos se utiliza el feedback para cambiar la conducta de los alumnos y dirigirla hacia el objetivo de aprendizaje, siendo de esta manera una información útil para el alumno y favoreciendo el proceso de enseñanza-aprendizaje. Estos datos están en la misma línea de otros estudios (Black \& Wiliam, 1998; Wiliam \& Leahy, 2015), en los que señalan que cuando el feedback se lleva a cabo de manera regular y con ánimo formativo se convierte en una estrategia clave para llevar a cabo un sistema de evaluación formativo efectivo.

Como ha podido comprobarse, los sistemas de evaluación que utilizan estos profesores estarían claramente situados en lo que se conocen como los sistemas de evaluación «alternativa», identificándose de forma clara con el modelo de EFyC y de EPA (López-Pastor, et al., 2013; MacPhail \& Murphy, 2017; Tolgfors \& Öhman, 2015). Diferentes estudios indican que su uso parece mejorar la calidad de los procesos de enseñanza y aprendizaje en EF en primaria (López-Pastor, 1999, 2006; Ní Chróinín \& Cosgrave, 2013). En todo caso, también existen estudios centrados en el impacto de la evaluación formativa en EF que parecen ofrecer resultados limitados (Hay, 2006; MacPhail \& Halbert, 2010; MacPhail \& Murphy, 2017).

Envarios estudios internacionales (Borghouts et al., 2016; Leirhaug \& MacPhail, 2015; Leirhaug \& Annerstedt, 2016; Ní Chróinín \& Cosgrave, 2013) puede comprobarse como es poco habitual que el profesorado de EF cumpla los criterios de la EPA(Leirhaug \& MacPhail, 2015; MacPhail \& Halbert, 2010; Tolgfors \& Öhman, 2015), incluso aunque la normativa del país así lo establezca; lo cual demuestra que el cambio educativo es un proceso complejo, y que no es suficiente con cambiar las leyes para que cambien las prácticas docentes del profesorado (Fullan, 2002). En este sentido, en la mayoría de los estudios sobre evaluación en EF parece que suele predominar la utilización del sistema tradicional, más orientado a obtener la calificación del alumnado a través de los test de condición física y/o habilidad motriz (López-Pastor, 1999; López-Pastor et al., 2013; MacPhail \& Murphy, 2017; Quitério et al., 2017), incluso aunque no aparezca en los objetivos (Borghouts et al, 2016), ni sea lo que establece la ley educativa (Leirhaug \& Annerstedt, 2016), o que se le relacione con la calidad de la EF (Rodríguez-Rodríguez et al., 2017).

Papel de la Formación Inicial del Profesorado en la realización o no de prácticas de Evaluación Formativa y Compartida y Evaluación para el Aprendizaje

Todos los maestros afirman haber recibido un modelo de evaluación tradicional durante su FIP (exámenes finales y calificación) salvo en un caso, que sí tuvo algunas experiencias de EFyC durante su FIP:

Cuando llegue a la escuela de magisterio,... según con que profesores. Algunos fueron los primeros con los que trabaje con este sistema de evaluación. No todos hacían evaluación formativa, pero algunos sí. La evaluación recibida (la mayoría tradicional) ha influido, pero al revés de lo habitual, en no gustarme y en animarme a buscar algo que sea diferente (E4).

Hetenido pocas experiencias relacionadas con la evaluación. Cuan- do empecé a trabajar en secundaria, reproduces lo qué has visto como alumno: conceptos, procedimientos y actitudes, con un instrumento específico para cada uno... Los alumnos ahora tienen otros intereses y tú tienes que adaptar tú evaluación y enseñanza (E2).

Sin embargo, todos los entrevistados han ido evolucionando hacia sistemas de EFyC a lo largo de su carrera profesional. La razón principal para el cambio tiene que ver con sus creencias y convicciones sobre cómo debe ser la educación, y sobre el efecto que ven que tiene la evaluación en los procesos de aprendizaje de su alumnado:

He recibido una evaluación tradicional ¿Por qué he decidido cambiar? Yo creo que, por coherencia, más por creencia casi que por conocimiento. Me pregunté: ¿Cómo queremos que aprendan nuestros chavales? Y una de las cosas que te replanteas es que, si el objetivo es que aprendan, y la evaluación es algo puntual, aislada de lo que estás dando, entonces tienes que buscar otras alternativas (E1).

La evaluación que he recibido es la tradicional y ha influido para cambiar, ya que no veía que los alumnos aprendieran (E3).

Los resultados encontrados muestran que la FIP parece haber influido poco o nada en la utilización de sistemas de EFyC por parte de los entrevistados. Sin embargo, si hay una persona que señala cómo ha influido positivamente el que algunos profesores, una minoría, llevaran a cabo sistemas de EFyC; lo que coincide con lo encontrado por Hamodí et al. (2017), sobre que los maestros valoran muy positivamente el haber tenido experiencias de EFyC durante su FIP, aunque hayan sido escasas y que les ha influido a la hora de trasferir dichas experiencias a su labor docente. Una explicación de ello se puede encontrar en la edad de los maestros entrevistados. Los estudios indican que cuando ellos realizaron su FIP, en la universidad española predominaban de forma casi absoluta los modelos de evaluación-calificación tradicionales (Zabalza, 2003). En cambio, estudios recientes demuestran que esto está cambiando de forma notable en las facultades encargadas de la FIP, especialmente desde 2010, de forma que actualmente en muchas facultades aproximadamente un tercio del profesorado ya utiliza sistemas de EFyC en FIP, otro tercio mantiene todavía los modelos tradicionales y otro tercio utiliza sistemas eclécticos (Palacios \& López-Pastor, 2013). Además, algunos estudios muestran que desde hace casi dos décadas existe una clara preocupación por implantar y desarrollar nuevos sistemas de evaluación en la FIP de EF, que podrían encuadrarse en los modelos denominados EFyC, EPA y EA (Fernández-Balboa, 2006; López-Pastor, 2006, 2008; López-Pastor \& Sicilia, 2017; Lorente y Kirk, 2013; Rodríguez-Aceituno \& Pina, 2018). Aunque también existen estudios que encuentran diferentes percepciones entre profesorado y alumnado sobre la evaluación recibida en FIP de EF (Martínez, Santos \& Castejón, 2017).

Una de las principales razones que parece llevar a los entrevistados a cambiar sus prácticas evaluativas son sus creencias educativas. Todos ellos tiene un discurso ideológico muy cercano a un marco de racionalidad práctico, el cual entra en contraposición al discurso que han recibido en su FIP, más cercano al marco de racionalidad técnico, predominante cuando ellos estudiaban (López-Pastor, 1999; López-Pastor, Monjas \& Pérez, 2003). Este «choque» ideológico es el principal precursor de que los entrevistados investigarán y buscarán información sobre prácticas evaluativas que encajarán mejor con su idiosincrasia. También pueden encontrarse interesantes estudios autobiográficos que explican porque algunos profesores de EF comienzan a aplicar sistemas de EFyC en sus aulas, en contra de formación recibida y de lo que era la tradición docente cuando acceden a la profesión (Córdoba, 2015; Córdoba et al, 2016; Molina \& López, 2017).

La Formación Permanente del Profesorado (FPP) ha tenido una evolución similar en todos los entrevistados. De una manera u otra, en un momento concreto de su carrera docente han conocido, encontrado y mostrado interés hacia modelos de EFyC. En la mayoría de los casos comenzó al formar parte de grupos de trabajo y/o de manera autodidacta (leyendo bibliografía, asistiendo a congresos, cursos, etc.).

Durante la carrera sólo hice un año de magisterio en Segovia y 
después me fui a hacer INEF. En el INEF, la formación recibida a nivel pedagógico tiene muchas lagunas y hace más lo que tú acabes leyendo.....y en mi caso, ha influido mucho en ese sentido, un grupo de trabajo que utiliza una EFyC(E2).

Leí la tesis de X sobre evaluación formativa y me llamo tanto la atención cómo argumentaba, el por qué hacía las cosas, que yo también quería justificar mi práctica docente y acabe haciendo la tesis sobre este tema (E4).

Los datos recogidos parecen indicar que la FPP es una de las piezas claves a la hora de conseguir que el profesorado de EF evolucione desde modelos de evaluación tradicional a modelos de EFyC:

«X» me dejó mucho material de cómo trabajar los PAT (proyecto de aprendizaje tutorado), lo apliqué en Sevilla y me pareció más coherente. Esto me abrió las miras a otros términos: «Evaluación formativa», «trabajo por proyectos»,... Para formarme: hablocon gente que tiene una filosofía parecida y veo que funciona, voy a algún congreso a conocer otras experiencias. La gente que hace formación son gente que se suele preocupar, tener buenas ideas y propuestas que te puedan ayudar (E1).

Cayó en mis manos un libro de evaluación formativa y de allíya me puse en contacto con el autor y hasta hoy (eso fue en el 2004). Así que, fundamentalmente era autodidacta, buscando en bibliografía. Además, he realizado algunos cursos de evaluación formativa y estoy en un grupo de trabajo que utiliza sistemas de EFyC (E3).

Cada uno de los entrevistados se ha ido formando de distintas maneras: cursos, congresos, lecturas, etc., pero, sobre todo, a través de diferentes actividades de FPP, en las que tienen un papel especialmente importante la participación en grupos de trabajo colaborativos y la asistencia a congresos profesionales. Las afirmaciones de la mayoría de los entrevistado parecen indicar que la FPP es especialmente relevante y potente cuando está ligada a grupos colaborativos de investigaciónacción, centrados en procesos de desarrollo profesional docente (Córdoba, 2015; Córdoba et al., 2016; López-Pastor et al., 2016; Lorente \& Kirk, 2013; Pedraza \& López-Pastor, 2015).

\section{Conclusiones}

Se confirma que todos los profesores entrevistados utilizan sistemas de EFyC y EPA en sus clases de EF en primaria y secundaria, resaltando las siguientes conclusiones:

1- Todos los profesores llevan a cabo un sistema de evaluación en base a los tres criterios establecidos: compartir con los estudiantes los objetivos de aprendizaje y los criterios de evaluación a utilizar en la UD o curso, implicar al alumnado en la evaluación de su propio aprendizaje y facilitar feedback a los estudiantes, que les ayude a entender que deben hacer para mejorar su aprendizaje.

2- Los profesores señalan las siguientes ventajas de aplicar estos sistemas de evaluación: se mejora el clima de aula y los alumnos son más conscientes y participes de los objetivos de aprendizaje; los procesos de autoevaluación, coevaluación y evaluación compartida favorecen el proceso de aprendizaje y regula los procesos de enseñanza y aprendizaje; el feedback regular y útil ayuda a que los alumnos se fijen más en los objetivos de aprendizaje y a reconducir las tareas en las que participan.

En los cuatro casos los sistemas de evaluación experimentados durante su FIP influyen inicialmente en sus primeras prácticas docentes. Sin embargo, una vez que se enfrentan a la realidad educativa parecen existir varios motivos que les inducen a evolucionar hacia sistemas deEFyC: (1) las creencias educativas de los entrevistados, cercanas a un marco de racionalidad práctico; y, (2) participar en actividades de formación permanente, como la asistencia a cursos y congresos, lectura de bibliografía relacionada con prácticas de EFyC y/o EPA y, sobre todo, la pertenencia a grupos de trabajo que trabajan con este tipo de sistemas de evaluación, más coherentes con sus creencias educativas.

La demanda de todos los entrevistados es clara, la FIP recibida relacionada con sistemas de EFyCy EPA ha sido escasa o, en la mayoría de los casos, inexistente, por lo que han decidido realizar una formación permanente relacionada con este ámbito. Por todo ello, este estudio puede ser de utilidad para que en las universidades españolas incluya suficientes contenidos y prácticas relacionados con sistemas EFyC y/o EPA durante la FIP de EF. Además, tanto la FIP como la FPP deberían tener un enfoque práctico, en el que primara el conocimiento de experiencias docentes útiles y contrastadas como buenas prácticas. También puede ser interesante para el profesorado de EF que desee conocer las vivencias de otros compañeros cuando llevan a cabo sistemas de EFyC y/o EPAen primaria y secundaria y las ventajas e inconvenientes de su implementación en el aula.

Como prospectiva, sería interesante seguir investigando la temática, ampliando la muestra de profesores de EF en primaria y secundaria y conociendo en profundidad sus razones para utilizar sistemas de EFyC y/o PEA. También puede ser relevante comprobar si en la FPP existe una demanda relacionada con los sistemas de EFyC y/o EPA.

\section{Referencias}

Baker, D. J. \& Zuvela, D. (2013). Feedforward strategies in the first-year experience of online and distributed learning environments. Assessment \& Evaluation in Higher Education, 38(6), 687-697. doi:10.1080/ 02602938.2012.691153.

Black, P. \& Wiliam, D. (1998). Assessment and classroom learning. Assessment in Education: principles, policy \& practice, 5(1), 7-74.

Borghouts, L., Slingerland, M. \& Haerens, L. (2016). Assessment quality and practices in secondary PE in the Netherlands. Physical Education and Sport Pedagogy, 1-17. DOI: 10.1080/ 17408989.2016.1241226.

Casbon, C. \& Spackman, L. S. (2005). Assessment for learning in Physical Education. Leeds, BAALPE.

Córdoba, C. (2015). La aventura de aprender: Relato autobiográfico del viaje a Ítaca de un docente reflexivo. Retos de la Actividad Física y el Deporte, 28, 285-290.

Córdoba, C., Carbonero, L., Sánchez, D., et al. (2016). Educación Física Cooperativa, formación permanente y desarrollo profesional. De la escritura colectiva a un relato de vida compartido. Retos de la Actividad Física y el Deporte, 29, 264-269.

Desrosiers, P., Genet-Volet, Y. \& Godbout, P. (1997). Teachers’ Assessment Practices Viewed Through the Instruments Used in Physical Education Classes. Journal of Teaching in Physical Education, 16(2), 211228.

Dulamã, M. E. \& Ilovan, O. R. (2016). How powerful is feedforward in university education? A case study in Romanian geographical education on increasing learning efficiency. Educational Sciences: Theory \& Practice (ESTP), Kuram ve Uygulamada Eðitim Bilimleri, 16(3), 827-848. doi: 10.12738/estp.2016.3.0392.

Fernández-Balboa, J. M. (2006). Dignity and democracy in the college classroom: The practice of self- evaluation, in R. A. Goldstein, ed. Useful theory: Making critical education practical. New York: Peter Lang Publishing.

Fraile-Aranda, A., López-Pastor, V. M., Castejón-Oliva, F. J. RomeroMartín, R. (2013). La evaluación formativa en docencia universitaria y el rendimiento académico del alumnado. Aula Abierta, 41(2), 23-34.

Fullan, M. (2002). Los nuevos significados del cambio en la educación. Barcelona: Octaedro.

Gibbs, G. \& Simpson, C. (2004). Does your assessment support your students' learning? Journal of Teaching and Learning in Higher Education 1, 3-31.

Goldsmith, M. (2008). Try feedforward instead of feedback. The Linkage Leader, 1-5.

Gomez, I. R., Del Valle, S., \& De la Vega Marcos, R. (2018). Revisión nacional e internacional de las competencias profesionales de los docentes de Educación Física. Retos. Nuevas tendencias en Educación Física, Deportes y Recreación, 34, 383-388. En: https:// recyt.fecyt.es/index.php/retos/article/view/58609

Hamodi, C., López-Pastor, V. M., López, A. T. (2017). If I experience formative assessment whilst at University will I put it into practice later as a teacher? Formative and shared assessment in Initial Teacher Education (ITE). European Journal of Teacher Education, 40(2), 171-190. doi:10.1080/02619768.2017. 1281909.

Hay, P. J. (2006). Assessment for learning in Physical Education. En: D. Kirk, D. MacDonald \& M. O'Sullivan. (Eds), The handbook of 
Physical Education (pp.312-325). London, England: Sage.

Herranz, M.; López-Pastor, V. M. (2014). ¿Es Viable Llevar a Cabo Procesos de Autoevaluación y Evaluación Compartida en Educación Física en la Etapa de Educación Primaria? Un Estudio de Caso Longitudinal. Revista de Educación Física, Renovar la teoría y la práctica, 133(30-1),11-18.

Herranz, M. \& López-Pastor, V.M. (2017). Perspectiva del alumnado sobre su participación en los procesos de evaluación formativa y compartida en educación física en primaria: un estudio de caso. EmásF. Revista digital de educación física, 48, 27-48. En: http:// emasf.webcindario.com/Perpectiva_del_alumnado sobre su_participacion en_los_procesos_de_evaluacion _formativa_y_compartida.pdf

Koen, K., Bitzer, E. M. \& Beets, P. (2012). Feedback or feedforward? A case study in one higher education classroom. Journal of Social Sciences, 32(2), 231-242.

Leirhaug, P. E. \& MacPhail, A. (2015). It's the other assessment that is the key: three Norwegian physical education teachers' engagement (or not) with assessment for learning. Sport, Education and Society, 20(5), 624-640. doi:10.1080/13573322.2014.975113.

Leirhaug, P. E. \& Annerstedt, C. (2016). Assessing with new eyes? Assessment for learning in Norwegian physical education. Physical Education and Sport Pedagogy, 21(6), 616-631. doi:10.1080/ 17408989.2015.1095871.

López, L. M. \& Gutiérrez, D. (2018). Contributions of the GPET to the GPAI: tactical context adaptation and game behaviour. Retos. Nuevas tendencias en Educación Física, Deportes y Recreación 34, 323-328. Recuperado de: https://recyt.fecyt.es/index.php/retos/ article/view/63794

López-Pastor, V. M. (1999). Prácticas de Evaluación en Educación Física:estudio de casos en primaria, secundaria y formación del profesorado. Valladolid, Universidad de Valladolid.

López-Pastor, V. M. (coord.) (2006). La Evaluación en Educación Física: Revisión de los modelos tradicionales y planteamiento de una alternativa: La evaluación formativa y compartida. Buenos Aires: Miño y Dávila.

López Pastor, V. M. (2008). Implementing a Formative and Shared Assessment System in Higher Education Teaching. European Journal of Teacher Education, 31(3), 293-311.

López Pastor, V. M., Monjas, R. et al. (2006). La Evaluación en Educación Física. Revisión de los modelos tradicionales y planteamiento de una alternativa: La Evaluación Formativa y Compartida. Retos. Nuevas tendencias en Educación Física, Deportes y Recreación, 10, 31-41. En: https://recyt.fecyt.es/index.php/ retos/article/view/35061

López-Pastor, V. M., Kirk, D., Lorente-Catalán, E., MacPhail, A. \& Macdonald, D. (2013). Alternative Assessment in Physical Education: A Review of International Literature. Sport, Education \& Society, 18(1), 57-76. doi:10.1080/13573322.2012.713860.

López-Pastor, V. M., Monjas, R., Manrique, J. C., Barba, J. J. \& González, M. (2008). Implicaciones de la evaluación en los enfoques de educación física cooperativa. El papel de la evaluación formativa y compartida en la necesaria búsqueda de coherencia. Cultura y Educación, 20(4), 457-477.

López-Pastor, V. M., Monjas, R. \& Pérez, D. (2003). Buscando alternativas a la forma de entender y practicar la educación física escolar. Barcelona: INDE

López-Pastor V. M. \& Pérez-Pueyo, Á. (Coord.) (2017). Evaluación formativa y compartida en educación: experiencias de éxito en todas las etapas educativas. León, España: Universidad de León.

López-Pastor, V. M., Ruano, C., Hernangómez, A., et al. (2016). Veinte años de formación permanente del profesorado, investigación-acción y programación por dominios de acción motriz. Retos, Nuevas tendencias en Educación Física, Deporte y Recreación, 29, 270279.

López-Pastor, V. M. \& Sicilia-Camacho, A. (2017). Formative and shared assessment in higher education. Lessons learned and challenges for the future. Assessment \& Evaluation in Higher Education, 42(1). doi: 10.1080/02602938.2015.1083535

Lorente, E., \& Kirk, D. (2013). Alternative democratic assessment in PETE: an action-research study exploring risks, challenges and solutions. Sport, Education and Society,18(1), 77-96.

MacPhail, A. \& Halbert, J. (2010). We had to do intelligent thinking during recent PE': Students' and teachers' experiences of assessment for learning in post-primary physical education. Assessment in Education, 17(1), 23-39.
MacPhail, A., \& Murphy, F. (2017). Too much freedom and autonomy in the enactment of assessment? Assessment in physical education in Ireland. Irish Educational Studies, 1-16.

Martínez, L. F., Santos, M. L. \& Castejón, F. J. (2017). Percepciones de alumnado y profesorado en Educación Superior sobre la evaluación en formación inicial en educación física. Retos. Nuevas tendencias en Educación Física, Deportes y Recreación, 32, 76-81. Recuperado de https://recyt.fecyt.es/index.php/ retos/article/view/52918/33289

Mintah, J. K. (2003). Authentic Assessment in Physical Education: Prevalence of Use and Perceived Impact on Students' Self-Concept, Motivation, and Skill Achievement. Measurement in physical education and exercise science, 7(3), 161-174.

Molina, M. \& López-Pastor, V. M. (2017). La transferencia de la Evaluación Formativa y Compartida desde la Formación Inicial del Profesorado de Educación Física a la práctica real en Educación Primaria. Infancia, Educación y Aprendizaje (IEYA), 3(2), 626-631.

Morgan, P. \& Hansen, V. (2007). Recommendations to improve primary school physical education: Classroom teachers' perspective. Journal of Educational Research 101(2), 99-111.

Murtagh, L. \& Baker, N. (2009). Feedback to feed forward: Student response to tutors' written comments on assignments. Practitioner Research in Higher Education 3(1), 20-28.

Ní Chróinín, D. \& Cosgrave, C. (2013). Implementing formative assessment in primary physical education: teacher perspectives and experiences. Physical Education and Sport Pedagogy, 18(2), 219233. doi:10.1080/17408989.2012.666787.

Oslin, J. L., Mitchell. S. A. \& Griffin, L. L. (1998). The game performance assessment instrument (GAPI): development and preliminary validation. Journal of Teaching in Physical Education, 17(2), 231243.

Pedraza-González, M. A. \& López-Pastor, V. M. (2015). Investigaciónacción, desarrollo profesional del profesorado de educación física y escuela rural. Revista Internacional de Medicina y Ciencias de la Actividad Física y el Deporte, 15(57), 1-16. doi:10.15366/ rimcafd2015.57.001.

Perrenoud, P. (2004). Diez nuevas competencias para enseñar. Barcelona, España: Graó

Quitério, A., Costa, J.,Martins, M., Martins, J., Onofre, M., Gerlach, E., Scheuer, C \& Herrmann, C. (2017). Educação Física: Avaliação das competências motoras em alunos de seis anos, do primeiro ano de escolaridade. Retos. Nuevas tendencias en Educación Física, Deportes y Recreación, 31, 259-263. Recuperado de https://recyt.fecyt.es/ index.php/retos/article/view/53500/32239

Richard, J. F. \& Godbout, P. (2000). Formative Assessment as an integral part of the teaching-learning process. Physical and Health Education Journal, 66(3), 4-9.

Rodríguez-Aceituno, P. F. \& Pina, F. H. (2018). Didáctica de la Motricidad en la formación de profesores de educación infantil. Retos. Nuevas tendencias en Educación Física, Deportes y Recreación, 34, 25-32. En: https://recyt.fecyt.es/index.php/retos/article/view/58416

Rodríguez-Rodríguez, F. J., Curilem, C., Berral, F. J. \& Almagià, A. A. (2017). Evaluación de la educación física escolar en Enseñanza Secundaria. Retos. Nuevas tendencias en Educación Física, Deportes y Recreación, 31. 76-81. Recuperado de https://recyt.fecyt.es/ index.php/retos/article/view/49097/31439

Romero-Martín, R., Fraile-Aranda, A., López-Pastor, V. M. \& CastejónOliva, F. J. (2014). The relationship between formative assessment systems, academic performance and teacher and student workloads in higher education. Infancia y Aprendizaje, 37(2), 1-17.doi:10.1080/ 02103702. 2014.918818.

Svennberg, L., Meckbach, J. \& Redelius, K. (2014). Exploring PE teachers’ 'gut feelings': An attempt to verbalise and discuss teachers' internalised grading criteria. European Physical Education Review 20(2): 199214.

Taras, M. (2005). Assessment Summative and Formative, some theoretical reflections, British Journal of Educational Studies, 53 (3), 466-478.

Tolgfors, B. \& Ohman, M. (2015). The Implications of Assessment for Learning in Physical Education and Health. European Physical Education Review 22(2), 150-166.

Wiliam D \& Leahy S (2015). Embedding Formative Assessment: Practical Techniques for K-12 Classrooms. West Palm Beach, FL: Learning Sciences International.

Zabalza, M. A. (2003). Competencias docentes del profesorado universitario. Calidad y desarrollo profesional. Madrid, España: Narcea. 